\title{
VIBRATIONS OF TETRAHEDRAL Co AND Cu CLUSTERS ON Cu(111) SURFACE
}

\author{
Sergey Eremeev $^{1}$, Svetlana Borisova ${ }^{1}$, Galina Rusina ${ }^{1}$, and Eugene Chulkov ${ }^{2,3}$ \\ ${ }^{1}$ Institute of Strength Physics and Materials Science, Siberian Branch, Russian Academy of \\ Sciences, pr. Akademichesky 2/4, Tomsk, 634021, Russia \\ 2 Donostia International Physics Center (DIPC), Paseo de Manuel Lardizabal, 4, 20018 San \\ Sebastián/Donostia, Spain \\ ${ }^{3}$ Depto. de Física de Materiales and Centro Mixto CSIS-UPV/EHU, Facultad de Ciencias \\ Químicas, Universidad del País Vasco/Euskal Herriko Unibertsitatea, Apdo.1072, 20018 San \\ Sebastián/Donostia, Spain
}

\section{eremeev@ispms.tsc.ru}

The epitaxial thin-film growth of $\mathrm{Co}$ on $\mathrm{Cu}$ substrates has been studied extensively during the last years. Because of the giant magnetoresistance observed in $\mathrm{Co} / \mathrm{Cu}$ multilayer systems and its sensitivity to the atomic-scale morphology, extensive experimental studies have been devoted to their growth. These studies has been revealed a variety of growth modes [1-6]. At the initial stages of growth some of the deposited $\mathrm{Co}$ atoms can exchange with $\mathrm{Cu}$ substrate atoms. This atomic exchange in conjunction with its coverage dependence leads to three different adatom species on the surface: substitutional $\mathrm{Co}$, on-surface $\mathrm{Co}$, and on-surface $\mathrm{Cu}$, which can form compact clusters [1,6]. Numerous theoretical investigations have also been performed to study atomic structure as well as energetic and magnetic properties in $\mathrm{Co} / \mathrm{Cu}$ system. Using density-functional theory calculations possible equilibrium structures for a monolayer coverage of $\mathrm{Co}$ on $\mathrm{Cu}(001)$ were analyzed [2]. Atomistic processes involved in the growth of $\mathrm{Co}$ on $\mathrm{Cu}$ surfaces were studied by employing static energy calculations and accelerated molecular dynamics simulations [7]. The interplay between structure and magnetic properties of small cobalt clusters on $\mathrm{Cu}$ surface were investigated by $a b$ initio and tightbinding calculations [8]. Despite important role that vibrational properties of small clusters play in understanding of many phenomena such as adsorbate diffusion, island and film growth significantly less attention was paid to study adsorbate and cluster vibrations on $\mathrm{Cu}$ surfaces. Recently vibrational properties of a single adatom [9-11] as well as of a dimer, and trimer of Co on low-index $\mathrm{Cu}$ surfaces $[11,12]$ have been thoroughly studied by using tight-binding second moment approximation interatomic interaction potentials. It was shown that structural and vibrational properties of the Co clusters strongly depend on the substrate orientation and the cluster size.

The simplest 3D clusters that can be formed in this system, tetrahedral $\mathrm{Co}_{4}$ and $\mathrm{Cu}_{4}$ clusters are considered in the present work. We study vibrational properties of these clusters on $\mathrm{Cu}(111)$ surface by using many-body interatomic potentials developed within tight-binding second moment approximation (TB-SMA) [13,14]. To determine the equilibrium atomic structure of free and supported clusters standard molecular dynamic technique based on the TB interaction potentials is applied. To simulate a semi-infinite $\mathrm{Cu}(111)$ surface with an adsorbed cluster a thin-film model of a two-dimensional periodic slab consisting of 31 atomic layers of $\mathrm{Cu}(111)$ with $5 \times 5$ supercell to exclude direct cluster-cluster interaction is used (see Fig. 1). The chosen thickness prevents interaction between two opposite surfaces of the copper film. The calculations of vibrational spectra are carried out by the dynamical matrix method. Diagonalizing the matrix gives the eigenfrequencies and the polarization vectors of vibrations. The local vibration densities of states were obtained by projecting these eigenmodes onto atoms of the cluster or the substrate in a given $(x+y$ or $z)$ direction. We also have done calculations of the equilibrium structure and vibrations of the free standing $\mathrm{Cu}_{2}$ and $\mathrm{Co}_{2}$ as well as $\mathrm{Cu}_{4}$ and $\mathrm{Co}_{4}$ clusters. The calculated $\mathrm{Co}_{2}$ and $\mathrm{Cu}_{2}$ bond lengths, binding energies, and the stretch mode frequencies are in close agreement with available experimental data. 
In Fig. 2 we show the calculated local density of states (LDOS) for the $\mathrm{Cu}_{4}$ (a) and $\mathrm{Co}_{4}$ (b) cluster atoms and for the first and second $\mathrm{Cu}(111)$ layer atoms. Analysis of equilibrium structure of the adsorbed tetramers shows that in the case of $\mathrm{Cu}_{4}$ the interaction with $\mathrm{Cu}(111)$ substrate leads to weakening $\mathrm{Cu}-\mathrm{Cu}$ bonds within cluster while for the Co cluster interaction with the substrate affects only the Co-Co bonds between atoms closest to the surface. In both cases the clusters lose a high symmetry tetrahedral shape, which they have in a free standing state. The interaction of the $\mathrm{Co}$ and $\mathrm{Cu}$ clusters with the substrate leads to vibrations which correspond to translational and rotational degrees of freedom of the free clusters. These frustrated translation and frustrated rotation modes are in-plane polarized and lying in a lowfrequency region. The frequencies of these modes in the Co cluster are $\approx 2 \mathrm{meV}$ higher due to the stronger $\mathrm{Co}-\mathrm{Cu}$ interaction in comparison with the $\mathrm{Cu}-\mathrm{Cu}$ one. The vibrational modes of the free $\mathrm{Cu}_{4}$ upon its adsorption on the $\mathrm{Cu}(111)$ surface mixe with $\mathrm{Cu}$ bulk phonons and become almost delocalized. Contrary to that, in the $\mathrm{Co}_{4}$ cluster on the surface the high frequency modes remain strongly localized and mixed with the nearest neighbor atoms vibrations only. Like vibrational modes of smaller $\mathrm{Co}$ clusters on low-index $\mathrm{Cu}$ surfaces, the highest frequency vibration of the $\mathrm{Co}_{4}$ cluster splits due to different interactions with certain groups of nearest neighbor atoms of the substrate.

\section{References:}

[1] M. T. Kief and W. F. Egelhoff, Phys. Rev. B 47 (1993) 10785.

[2] R. Pentcheva and M. Scheffler, Phys. Rev. B 61 (2000) 2211.

[3] J. Fassbender, R. Allenspach, and U. Dürig, Surf. Sci. 383 (1997) L742.

[4] U. May, J. Fassbender, and G. Güntherodt, Surf. Sci. 377-379 (1997) 992.

[5] T. Bernhard, R. Pfandzelter, and H. Winter, Nucl. Instrum. Methods Phys. Res. B 203 (2003) 111.

[6]F. Nouvertné, U. May, M. Bamming, A. Rampe, U. Korte, G. Güntherodt, R. Pentcheva, and M. Scheffler, Phys. Rev. B 60 (1999) 14382.

[7] Radu A. Miron and Kristen A. Fichthorn, Phys. Rev. B 72 (2005) 035415.

[8] S. Pick, V. S. Stepanyuk, A. L. Klavsyuk, L. Niebergall, W. Hergert, J. Kirschner, and P. Bruno, Phys. Rev. B 70 (2004) 224419.

[9] Kai Liu and Shiwu Gao, Phys. Rev. Lett. 95 (2005) 226102.

[10]Kai Liu and Shiwu Gao, Phys. Rev. B 74 (2006) 195433.

[11] S. D. Borisova, S. V. Eremeev, G. G. Rusina, V.S. Stepanyuk, P. Bruno, and E. V. Chulkov, Phys. Rev. B 78 (2008) 075428.

[12] S.D. Borisova, G.G. Rusina, S.V. Eremeev, and E.V. Chulkov, Phys. Solid State 51 (2009) 1271.

[13] N. A. Livanov, V. S. Stepanyuk, W. Hergert, D. I. Bazhanov, P. H. Dederichs, A. Katsnelson, C. Massobrio. Phys. Rev. B 61 (2000) 2230.

[14] V. S. Stepanyuk, D. V. Tsivline, D. I. Bazhanov, W. Hergert, and A. A. Katsnelson, Phys. Rev. B 63 (2001) 235406.

\section{Figures:}

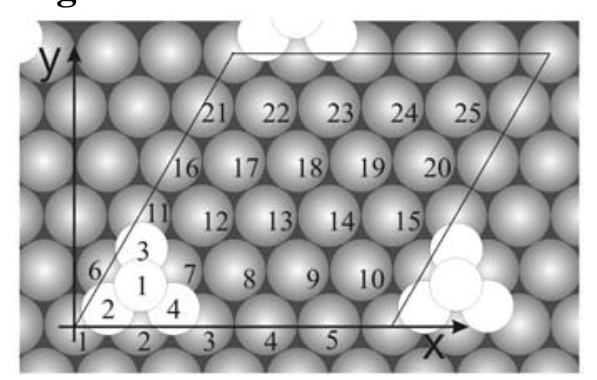

Figure 1 The atomic structure for adsorbed $\mathrm{Cu}_{4}\left(\mathrm{Co}_{4}\right)$ cluster on the $\mathrm{Cu}(111)$ surface. The computational $5 \times 5$ supercell is indicated by a rhombus, $x=[1 \overline{10}]$ and $y=[11 \overline{2}]$.
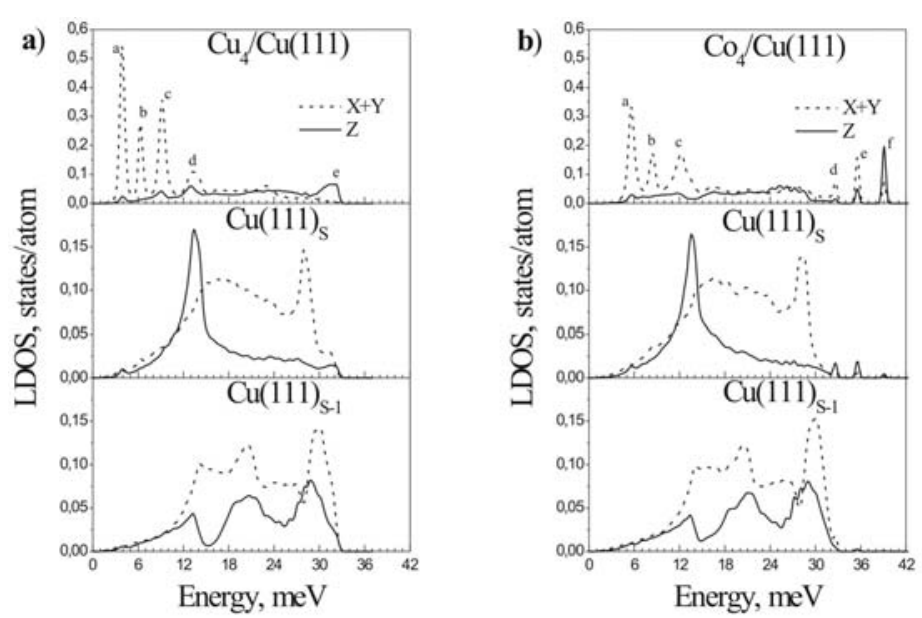

Figure 2 Local density of vibrational states for a tetrahedral clusters: a) $\mathrm{Cu}_{4}$ and b) $\mathrm{Co}_{4}$ as well as for the $\mathrm{Cu}(111)$ surface (S) and subsurface (S-1) atoms. 